\title{
Popliteal Artery Pseudoaneurysm after Anterior Cruciate Ligament Re-Revision Using a Rigidfix Cross Pin
}

\author{
Gwang Chul Lee, MD, Dong Hwi Kim, MD, and Sung-Hae Park, MD \\ Department of Orthopaedic Surgery, Chosun University Hospital, Gwangju, Korea
}

\begin{abstract}
Popliteal artery injury is a very rare complication of anterior cruciate ligament (ACL) reconstruction. The authors experienced a case of popliteal arterial pseudoaneurysm after re-revision of ACL reconstruction using Rigidfix for femoral tunnel fixation. Pseudoaneurysm was detected in knee magnetic resonance imaging, which caused pain, limit of motion, common peroneal nerve palsy, leg swelling and symptoms similar to compartment syndrome. After excision and re-anastomosis of the popliteal artery using a greater saphenous vein graft, all symptoms were resolved within 3 months except for common peroneal nerve palsy. So we report on this case with a review of the literature.
\end{abstract}

Keywords: Knee, Anterior cruciate ligament, Popliteal artery, Psedoaneurysm

Injury of the popliteal artery from knee arthroscopy is uncommon and few cases have been reported after anterior cruciate ligament (ACL) reconstruction ${ }^{1-5)}$. We present a case of popliteal artery injury accompanying pseudoaneurysm after use of Rigidfix cross pin system (DePuy Mitek Inc., Raynham, MA, USA) for femoral tunnel fixation using a transtibial technique during rerevision of ACL reconstruction with a review of the literature.

\section{Case Report}

A 29-year-old male patient who complained of pain and swelling around the popliteal area and symptoms of common peroneal nerve palsy in the right lower leg was referred to our service. The patient had a medical history of re-revision of ACL

Received July 15, 2013; Revised October 4, 2013;

Accepted December 12, 2013

Correspondence to: Gwang Chul Lee, MD

Department of Orthopaedic Surgery, Chosun University Hospital,

365 Pilmun-daero, Dong-gu, Gwangju 501-717, Korea

Tel: +82-62-220-3147, Fax: +82-62-226-3379

E-mail: smileleekci@gmail.com

This is an Open Access article distributed under the terms of the Creative Commons Attribution Non-Commercial License (http://creativecommons.org/licenses/by-nc/3.0/) which permits unrestricted non-commercial use, distribution, and reproduction in any medium, provided the original work is properly cited. reconstruction using a transtibial technique for femoral tunnel in the right knee 6 weeks ago in another hospital. The initial ACL reconstruction was performed 9 years ago with an Achilles tendon allograft after the patient had injured his knee during a soccer game. Revision was performed four years ago with an Achilles tendon allograft under the diagnosis of re-ruptured ACL that occurred during a basketball game. The patient's past history revealed popliteal fossa pain that started from the night of the 3rd operation (re-revision ACL reconstruction). The pain became aggravated on the 2 nd postoperative day, sudden swelling of the popliteal fossa was noted and symptoms of common peroneal nerve palsy developed. The surgeon who had performed the rerevision surgery could palpate the dorsalis pedis and posterior tibial arterial pulses and concluded that the patient had intact circulation and that the paralysis could be the result of inadequately long post-operative leg splinting. The patient was required to rest for 6 weeks. With no sign of pain and swelling subsiding, the patient was referred to our hospital.

On arrival, the dorsalis pedis and posterior tibia arterial pulses were palpated. Vital signs, including blood pressure, were within normal limits, and the results of blood gas analysis were normal. Dorsiflexion power of the right ankle was observed as grade 1-2 with $80 \%$ sensory loss compared to the contralateral limb. Ankle brachial index and toe pressure were not measured before surgery. Magnetic resonance imaging (MRI) and computed tomowww.jksrr.org 


\section{Lee et al. Popliteal Artery Pseudoaneurysm after Anterior Cruciate Ligament Re-Revision}

graphic (CT) angiography were performed for evaluation.

MRI findings showed abundant hematoma in the popliteal fossa and CT angiogram showed pseudoaneurysm of the popliteal artery and leakage of administered dye at the level just superior to the medial and lateral superior genicular artery (Fig. 1). From the MRI scan, the authors observed a trace of drilling track, presumably for Rigidfix insertion, which extended from anterior to posterior. The distal end of the drilling track was at the site of popliteal injury (Fig. 2). We believe that this drilling may have caused the arterial injury.

Symptoms similar to those of compartment syndrome, such as swelling, pain of the lower limb, and common peroneal nerve palsy and abundant hematoma on MR images are the reasons why we decided that decompression and exploration are required based on the result from the medical check-up.

Emergency surgical exploration was performed in the prone position through an $\mathrm{S}$ popliteal incision. Intraoperative findings revealed a hematoma measuring $8 \times 8 \mathrm{~cm}$ around the popliteal artery, which showed abundant adhesion with adjacent soft tissue and muscles. Segmental arterial injury was observed just superior to the level of the medial and lateral superior genicular artery.

Microscopic evaluation showed evidence of two perforating injuries and one peripheral injury (Fig. 3). A greater saphenous vein autograft was harvested from the ipsilateral limb and a reanastomosis procedure was performed (Fig. 4A).

Aspirin (Bayer, Leverkusen, Germany) and Cilostazol (Ple-
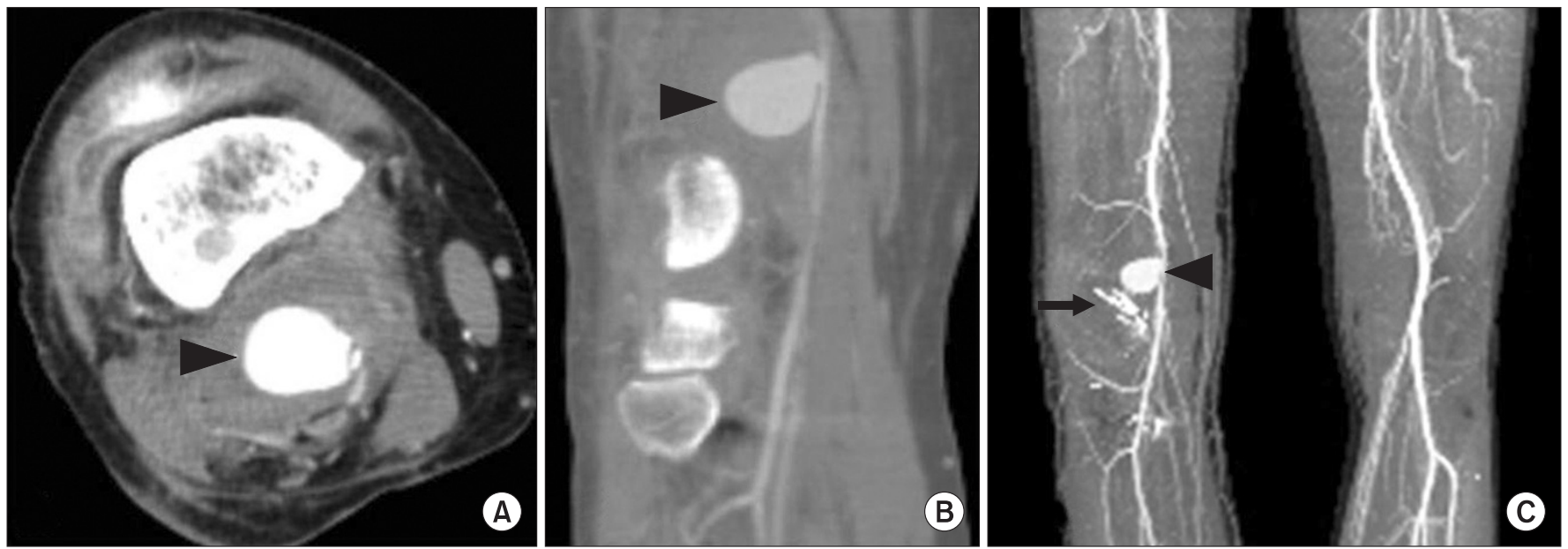

Fig. 1. Computed tomographic (CT) angiograms showing pseudoaneurysm (arrow head). (A) Axial view. (B) Coronal view. (C) CT arteriogram showing leakage of administered dye (arrow) at the level immediately superior to the medial and lateral superior genicular artery.
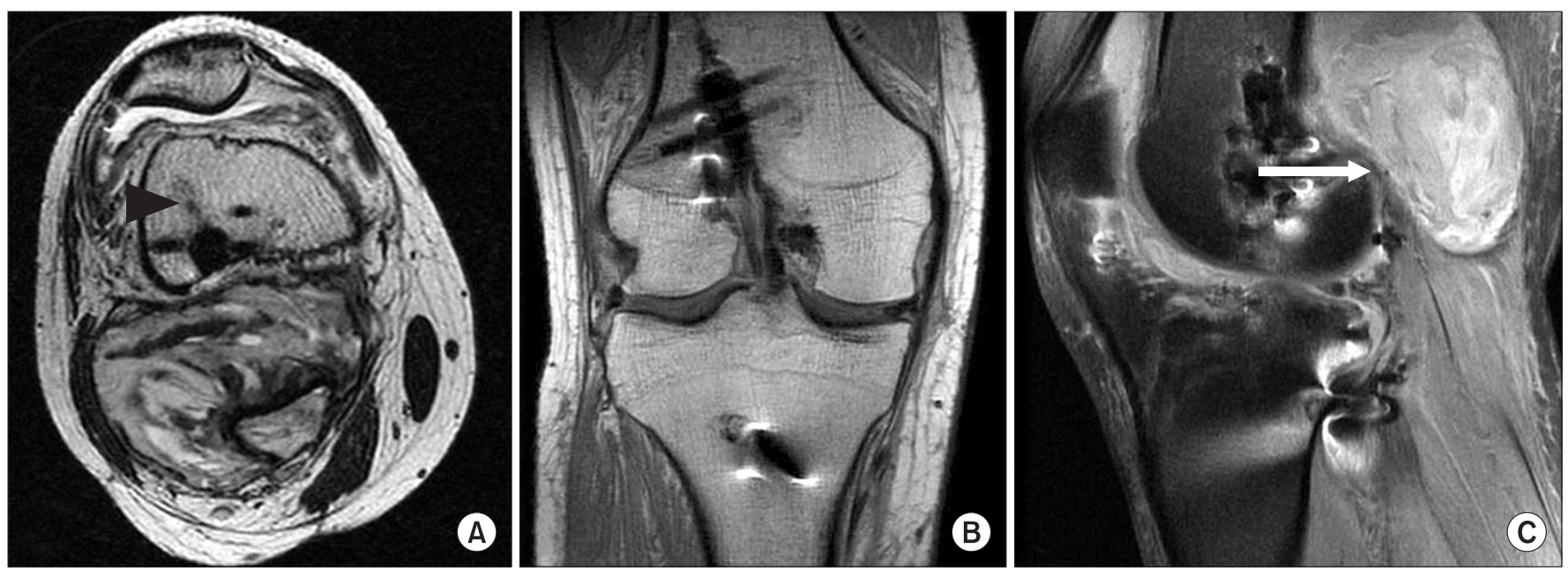

Fig. 2. (A) Axial magnetic resonance imaging (MRI) showing scars (arrow head) resulting from drilling for Rigidfix in an anterior-posterior direction around the femoral tunnel. (B) Coronal MRI showing the position of the anterior cruciate ligament femoral tunnel and the final position of Rigidfix. (C) Sagittal MRI showing a large hematoma (arrow) around the popliteal area. 


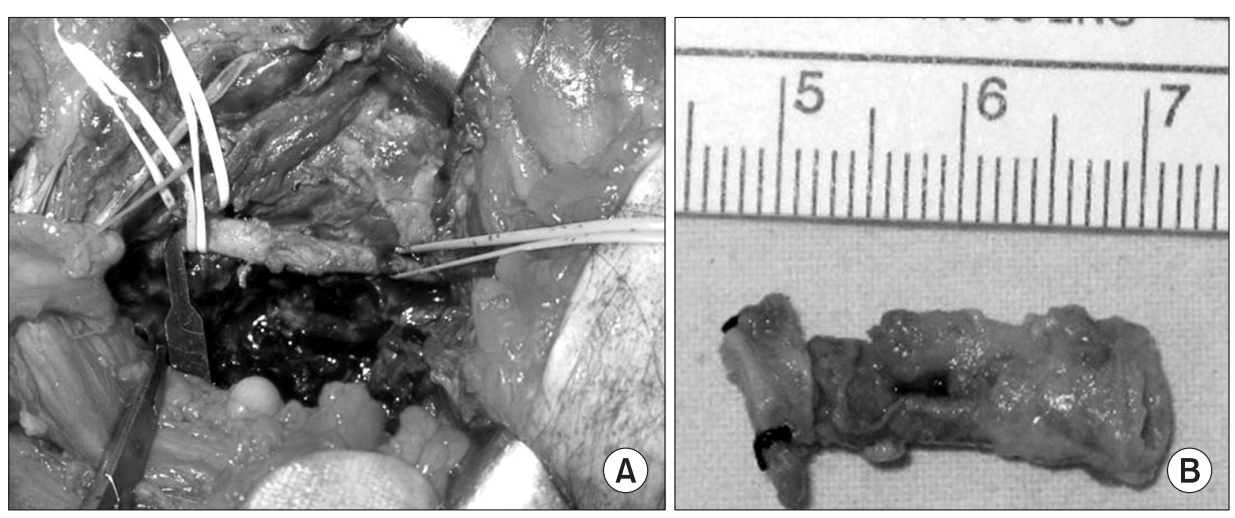

Fig. 3. Intraoperative photographs. (A) Intraoperative photograph showing the popliteal artery lacerated segmentally from immediately above the level of the medial and the lateral superior genicular artery. (B) Resection specimen of the segmentally lacerated popliteal artery.
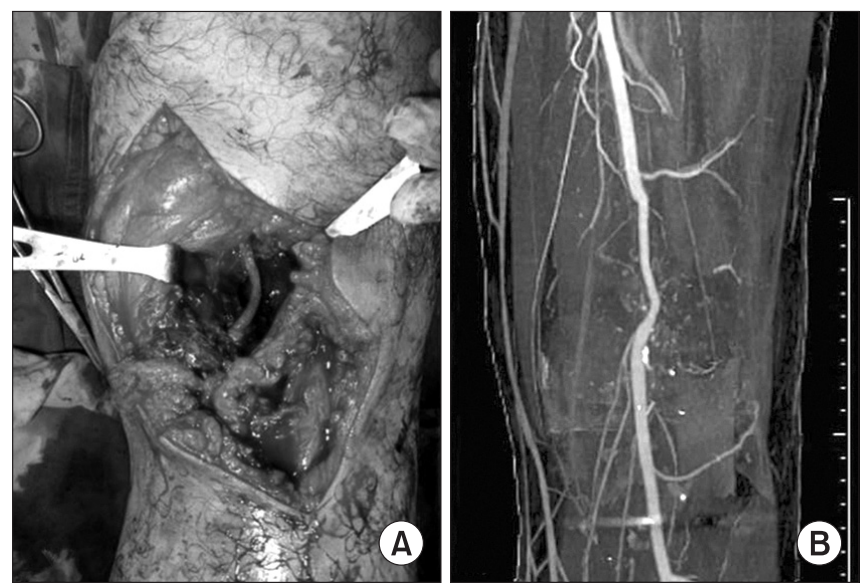

Fig. 4. Images showing re-established circulation after re-anastomosis of the popliteal artery with a greater saphenous vein graft. (A) Intraoperative photograph taken after re-anastomosis using a greater saphenous vein graft. (B) Computed tomography arteriogram obtained on postoperative day one.

taal; Otsuka, Tokyo, Japan) were administered for postoperative thrombo-prophylaxis. Ankle brachial index and toe pressure, compared to the contralateral limb, showed normal results on the third day after arterial re-anastomosis surgery. Follow-up $\mathrm{CT}$ angiograms obtained on the first postoperative day (Fig. 4B) showed intact circulations. Then, the patient was transferred to another hospital on patient demand.

Swelling and pain around the popliteal fossa were completely resolved and range of knee motion was restored three months after arterial re-anastomosis. However, the common peroneal nerve palsy detected by advanced Tinel's sign was partially improved and dorsiflexion power of the right ankle was still not improved.

\section{Discussion}

Vascular complications after arthroscopic ACL reconstruction have been rarely reported ${ }^{1-4)}$. Most of the previous publications documented direct injury to the popliteal artery, resulting in a fatal outcome or amputation of the limb. However, if blood circulation distal to the injured popliteal artery is maintained, favorable treatment results can be obtained despite a delayed diagnosis ${ }^{1,3)}$. In our case, knee pain and symptoms of common peroneal nerve palsy were attributed to the popliteal artery injury and the peripheral circulation was maintained for 6 weeks. It has not been clearly established whether common peroneal nerve palsy after ACL reconstruction is caused by mass effect of hematoma or iatrogenic injury. However, it seems more reasonable that the mass effect was the cause in our case since there was some improvement after the evacuation of hematoma.

Due to its convenience, superior biomechanical character, and favorable clinical outcome, the Rigidfix cross pin for femoral fixation of the ACL has recently become a popular choice ${ }^{6}$. However, Chang et al. ${ }^{6}$ reported the Rigidfix cross pin can be close to the popliteal artery when it is inserted in an anterior to posterior direction in a cadaveric study, thereby warning possible injury to the popliteal artery during pin insertion. In our case, a drill tip for the Rigidfix cross pin inserted from anterior to posterior caused a segmental perforation injury that could have resulted in a fatal outcome. In the re-revision operation, the widened femoral tunnel allowed inadequate placement of the Rigidfix's femoral rod, which may have caused twisting motion of the rod during drilling in the tunnel. In addition, the thin posterior cortex during drilling may have tricked the surgeon into redirecting the drill tip multiple times, which may also have contributed to the injury. For these reasons, we recommend, in cases where femoral tunnel widening is present, other fixation methods than Rigidfix cross pin system in re-revision ACL reconstruction.

From this experience, we recommend if the patient has symptoms of pain in the popliteal area and unexplainable paralysis following arthroscopic ACL reconstruction, a popliteal artery injury should be suspected even when the posterior tibial artery or the 


\section{Lee et al. Popliteal Artery Pseudoaneurysm after Anterior Cruciate Ligament Re-Revision}

dorsalis pedis artery can be palpated. Therefore, it is advised to perform angiography or angio-CT for prompt evaluation of the popliteal artery when an arterial injury is suspected and, if indicated, immediate surgical exploration.

\section{Conflict of Interest}

No potential conflict of interest relevant to this article was reported.

\section{References}

1. Aldridge JM 3rd, Weaver JP, Mallon WJ. Avulsion of the middle genicular artery: a previously unreported complication of anterior cruciate ligament repair: a case report. Am J Sports Med. 2002;30:748-50.

2. Janssen RP, Sala HA. Embolism of the popliteal artery after anterior cruciate ligament reconstruction: a case report and literature review. Knee Surg Sports Traumatol Arthrosc. 2007;15:1449-51.

3. Evans JD, de Boer MT, Mayor P, Rees D, Guy AJ. Pseudoaneurysm of the medial inferior genicular artery following anterior cruciate ligament reconstruction. Ann R Coll Surg Engl. 2000;82:182-4.

4. Janssen RP, Scheltinga MR, Sala HA. Pseudoaneurysm of the popliteal artery after anterior cruciate ligament reconstruction with bicortical tibial screw fixation. Arthroscopy. 2004;20:E4-6.

5. Furie E, Yerys P, Cutcliffe D, Febre E. Risk factors for arthroscopic popliteal artery laceration. Arthroscopy. 1995;11:324-7.

6. Chang CB, Yoo JH, Chung BJ, Seong SC, Kim TK. Oblique femoral tunnel placement can increase risks of short femoral tunnel and cross-pin protrusion in anterior cruciate ligament reconstruction. Am J Sports Med. 2010;38:1237-45. 